\title{
Giant atherosclerotic ascending aortic aneurism
}

\author{
Gregoriana Zanini · Alessandra Pelati · \\ Monica Bortolotti • Andrea Tironi • \\ Gian Franco Pasini
}

Received: 11 September 2009/Accepted: 3 November 2009/Published online: 3 December 2009

(C) SIMI 2009

\section{Introduction}

Ascending aorta aneurysms are commonly seen in patients with cardiovascular risk factors such as poorly treated hypertension, and hyperlipidemia (as a consequence of inflammation) in patients with a bicuspid aortic valve or in connective tissue disease or multisystem vasculitis disorders. Aortic ectasia is also described in patients undergoing valve replacement and in patients with an aortic stenosis; a poststenotic aneurysm formation can occur. Toxic substances such as cocaine and amphetamines can also lead to aortic wall thinning and aneurysm formation. An important cause of pseudo-aneurysm formation is related to trauma [1].

Hypertension, wall thinning and aortic enlargement are the most important factors in increasing wall stress, and lead to aortic rupture or dissection. Smoking increases the growth rate by $15-20 \%$. Aortic diameter is a marker of risk.

Aortic aneurysm is silent as long as there are no complications [2]. Aortic regurgitation is a typical consequence of ascending aortic aneurysm formation caused by an aortic ring dilatation with or without valve degeneration; aortic regurgitation may even be the first sign of the aneurysm formation [3].

Elective surgery has been recommended for aneurysm of the ascending aorta beyond $50 \mathrm{~mm}$, in patients with a bicuspid

G. Zanini $(\bowtie) \cdot$ A. Pelati · M. Bortolotti · G. F. Pasini

Section of Cardiology Ospedale di Gavardo,

Gavardo, Brescia

e-mail: gregoriana77@libero.it

\section{A. Tironi}

Section of 2nd Anatomy Pathology,

University Study of Brescia, Spedali Civili di Brescia, Brescia aortic valve or an increase of $0.5 \mathrm{~cm} /$ year, $55-60 \mathrm{~mm}$ in atherosclerotic and connective tissue diseases [4].

\section{Case report}

A 61-year-old woman was referred for an echocardiogram because of an ascending aorta aneurysm image discovered on a chest X-ray study performed for persistent cough (Fig. 1). She felt well except for the cough; there were no historical clues and no cardiovascular risk factors.

A transthoracic echocardiogram showed a giant ascending aorta aneurysm not involving the sinus of Valsalva, but beginning at the sinutubular junction $(75 \mathrm{~mm})$ and proceeding to the proximal ascending aorta $(82 \mathrm{~mm})$ up to the origin of the innominate artery $(48 \mathrm{~mm})$ (Figs. 2, 3). Severe aortic insufficiency in the tricuspid aortic valve was associated with initial signs of left ventricular enlargement, but a preserved ejection fraction. The patient was immediately admitted to our cardiology department and submitted to an computerized axial tomography (CT scan) that confirmed the echocardiography (Fig. 4). During the stay in hospital we screened for immunological and connective tissue disease. Serological infective markers were all negative.

The patient was scheduled for cardiac surgery. No significant atherosclerosis was detected at coronary angiography.

During surgery, aortic valve substitution with a mechanical prosthesis (Sorin Overline number 24) and aortic ascending substitution with Vascutek tube (number 34) were performed with optimal results.

The clinical course was uncomplicated, and the patient was discharged after 1 week in good condition. After 2 months of follow up she is in good health. 


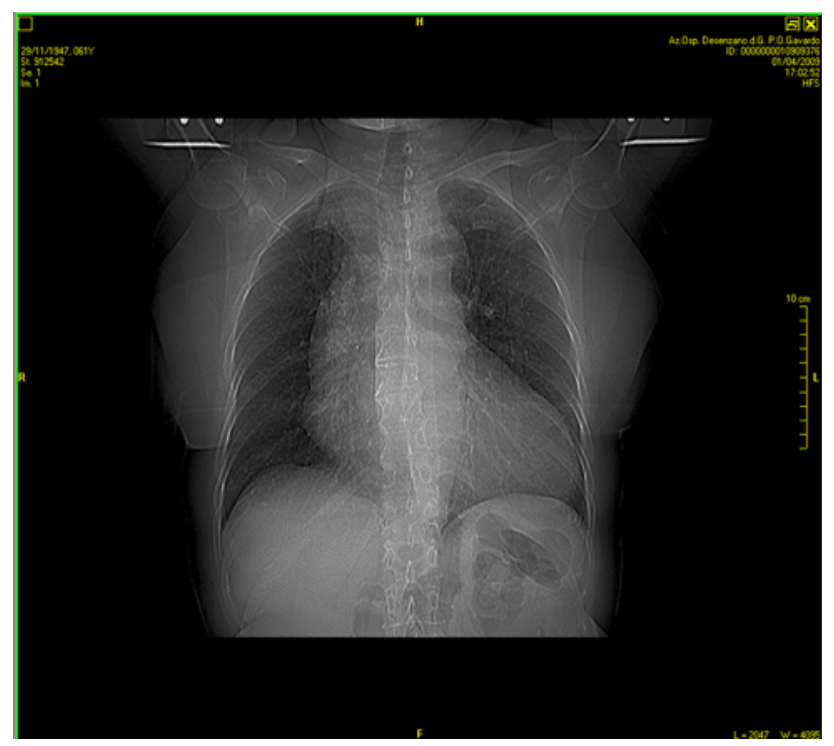

Fig. 1 X-ray showing an enlargement of cardiac silhouette

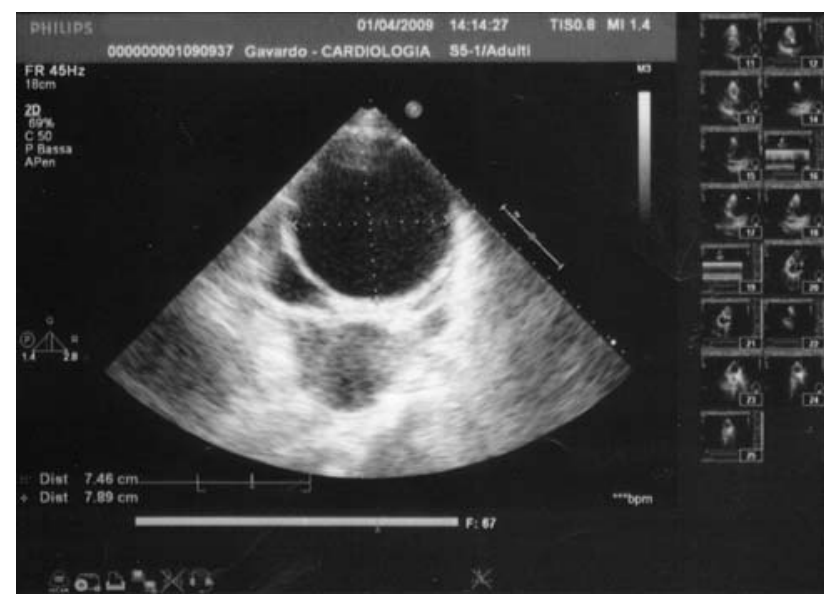

Fig. 2 Transthoracic echocardiogram: ascending aorta dimension in short axis

Histopathological finding of operating specimen of ascending aorta revealed an atherosclerotic aneurysm (Figs. 5, 6).

\section{Discussion}

Ascending aorta aneurysms are rare with an increased incidence in patients with atherosclerotic disease, connective tissue disease or a bicuspid aortic valve. There is no particular gender distribution, but atherosclerotic aneurysm is more frequent in men while genetic disorders predominantly affect women.

The aortic diameter is a risk marker of rupture but there are described cases of rupture even with aneurysms of

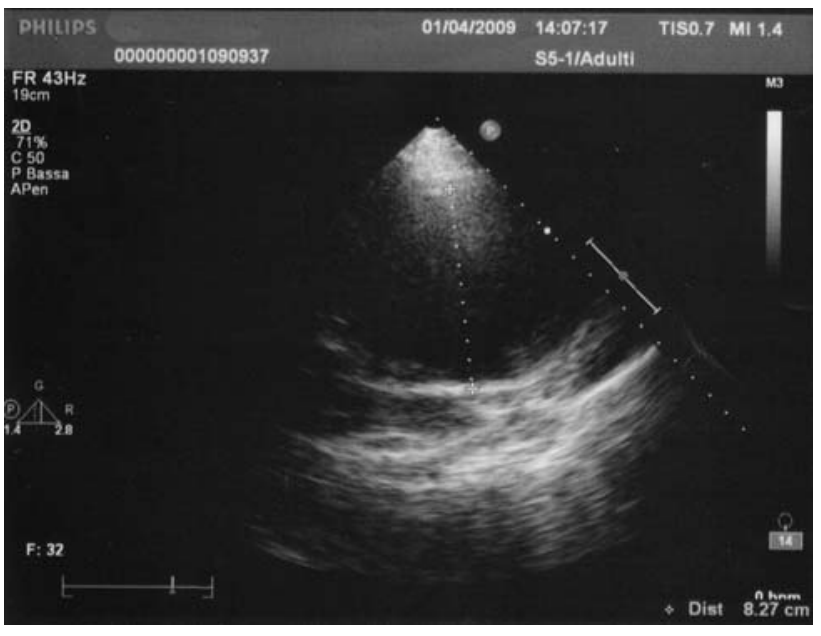

Fig. 3 Transthoracic echocardiogram: ascending aorta dimension in long axis

small dimension. Guidelines recommend elective surgery for aneurysm of the ascending aorta beyond $45 \mathrm{~mm}$ in a bicuspid aortic valve, and $55 \mathrm{~mm}$ in atherosclerotic and connective tissue diseases. Other complications of atherosclerotic aneurysm are embolization of atherosclerotic materials and thrombosis [5].

In our case, the giant ascending aorta aneurysm was detected in a middle aged women without any risk factor for aortic dilatation in particular without connective disease, but with the histological findings of an atherosclerotic aneurysm. There was not family history of aneurysm or hyperlipidemia. Despite the severe aortic insufficiency, the patient was asymptomatic.

In our patient, the enormous dimension of the ascending aorta fortunately did not lead to a catastrophic event such as a rupture.

Echocardiographic examination and CT scan play an essential role in the diagnosis of the patients affected by aortic aneurysm and aortic valve insufficiency (such as in our case).

The treatment of choice is surgical intervention. Cardiac surgery should be performed promptly after diagnosis in order to prevent complications such as aortic dissection, heart failure and sudden death. The intra-operative mortality rate in elective surgery is low $(1.5 \%)$ and the shortand long-term prognoses are good. Long-term problems can arise from anticoagulation (thromboembolism, valve thrombosis, haemorrhage) and endocarditis.

\section{Conclusion}

Aortic aneurysms are silent life threatening diseases. They can lead to serious complications and even sudden death in asymptomatic patients. In our case the risk was really great, 


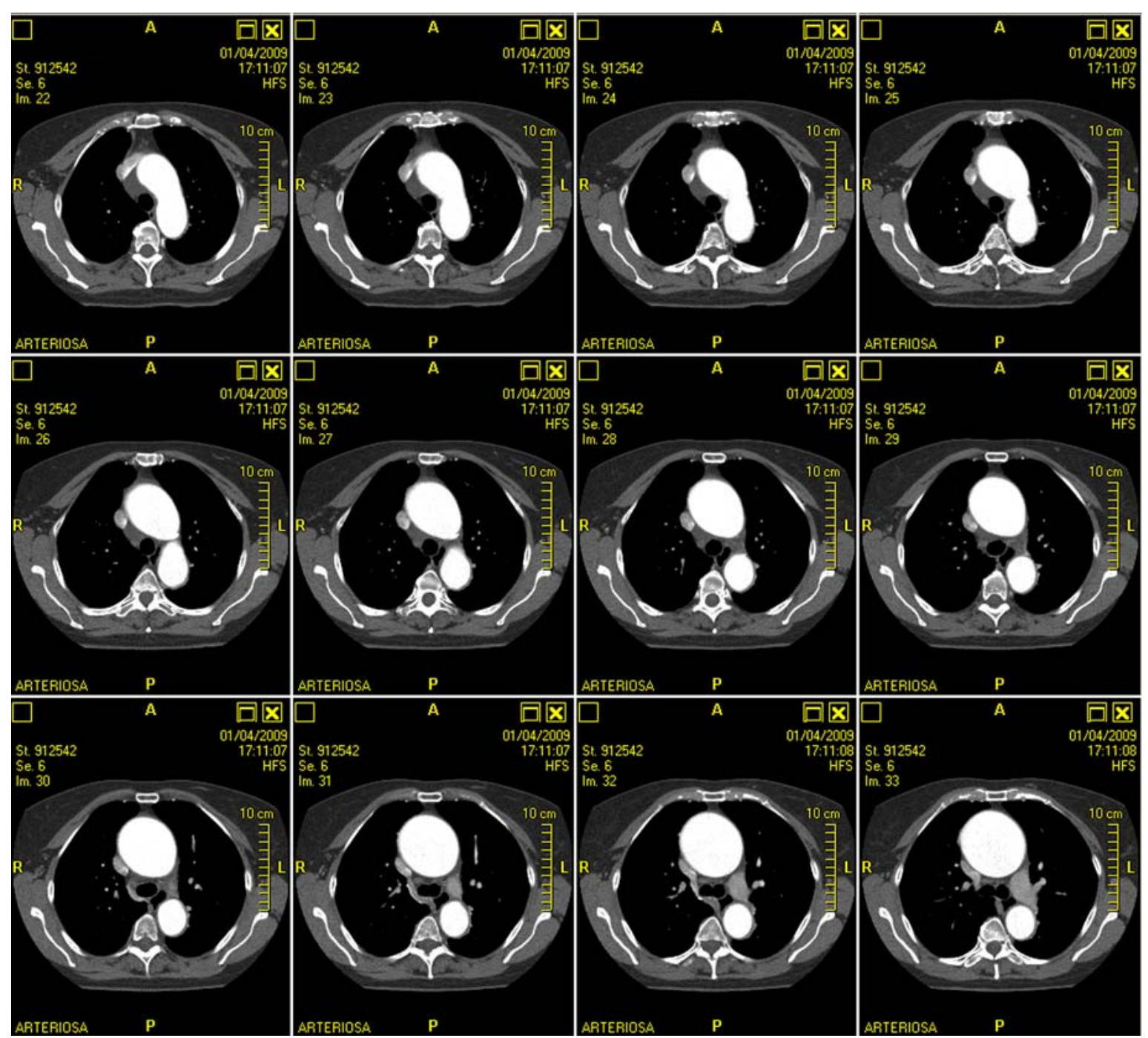

Fig. 4 Computerized axial tomography

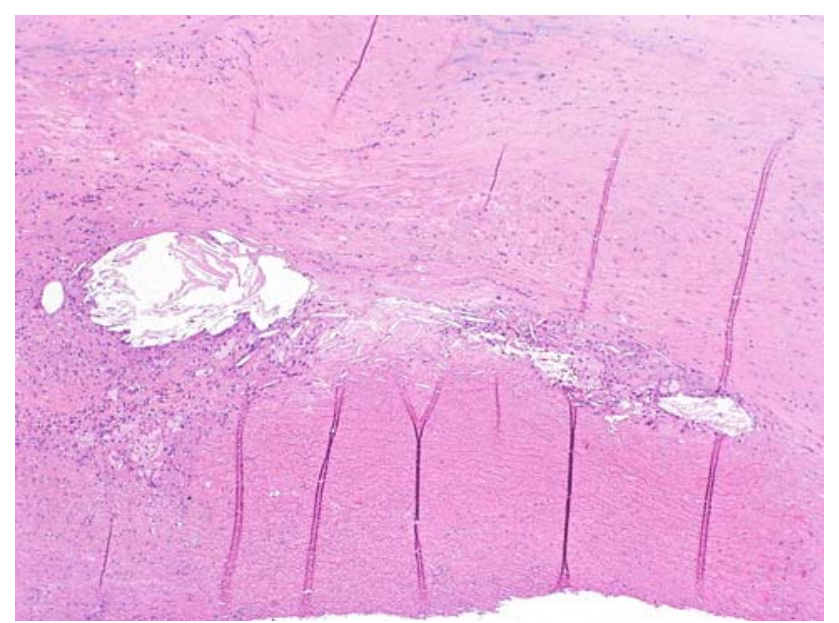

Fig. 5 Histologic section of inner aortic wall with atherosclerosis. $\mathrm{H} \& \mathrm{E}, \times 4$

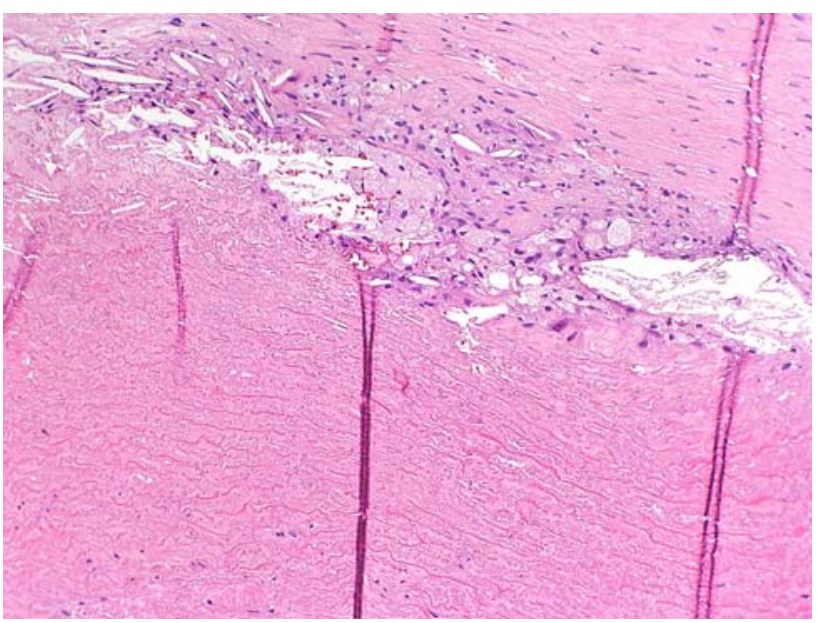

Fig. 6 Histologic section of aortic wall atherosclerosis. H\&E, $\times 10$ 
and the misleading symptom of cough was the life line for our patient. The enormous dilatation was unexpectedly related to atherosclerotic pathology in a patient without atherosclerosis risk factor.

Consent: "Written informed consent was obtained from the patient for publication of this case report and accompanying images.

Conflict of interest statement The authors declare that they have no conflict of interest related to the publication of this manuscript.
2. Elefteriades JA (2002) Natural history of thoracic aortic aneurysm: indications for surgery, and surgical versus non surgical risks. Ann Thorac Surg 74:S1877-S1880

3. Alegret JM, Vernis JM, Palazón O, Borràs X, Duran I (2005) Factors related to aortic regurgitation in the presence of a dilated aortic root. Am J Cardiol 95(3):417-420

4. Guntheroth WG (2008) A Critical Review of the American College of Cardiology/American Heart Association Practice Guidelines on bicuspid aortic valve with dilated ascending aorta. Am J Cardiol 102(1):107-110

5. Catapano Minotti G, Corsonello A, Guadalupi G, Antonelli Incalzi R. A thrombotic snake in the thoracic aorta. Intern Emerg Med. 2009. doi:10.1007/s11739-009-0288-6

\section{References}

1. Erbel R, Eggebrecht H (2006) Aortic dimension and the risk of dissection. Heart 92:137-142 